\title{
SOLUTION TO WEIGHTED NON-LOCAL FRACTIONAL DIFFERENTIAL EQUATION
}

\author{
Mohammed Mazhar-Ul-Haque ${ }^{1}$, Tarachand L. Holambe ${ }^{2}$, Govind P. Kamble ${ }^{3}$ \\ ${ }^{1}$ Dr. B.A.M. University \\ Aurangabad, Maharashtra, INDIA \\ ${ }^{2}$ Department of Mathematics \\ Kai Shankarrao Gutte ACS College \\ Dharmapuri, Beed, Maharashtra, INDIA \\ ${ }^{3}$ Department of Mathematics \\ P.E.S. College of Engineering \\ Nagsenvana, Aurangabad, (M.S.) INDIA
}

\begin{abstract}
In this paper we prove the nature and existence of the solutions for a weighted nonlinear fractional differential equation with nonlocal condition. Given a bounded interval $J=(0, T]$ of the real line $\mathbb{R}$ for some $T>0$ and $T<\infty$, we consider the fractional differential equation

$$
\begin{aligned}
A_{0} v(t)+\sum_{i=1}^{n} A_{i} D^{\beta_{\mathrm{i}}}(v(t) u(t)) & =D^{\alpha}(v(t) u(t)), \\
\lim _{t \rightarrow 0^{+}} t^{1-\alpha} v(t) u(t) & =\sum_{j=1}^{m} a_{j} u\left(\tau_{j}\right),
\end{aligned}
$$

where $D^{\alpha}$ and $D^{\beta_{\mathrm{i}}}$ are Riemann Liouville fractional derivatives of order $0<\alpha, \beta_{i} \leq 1$.

Under some assumptions the nonlocal weighted Cauchy type fractional differential equation and result on its solution will be discussed in nonlinear fractional differential equation.

AMS Subject Classification: 26A33, 34K37, 34A08, 45E10, 47H10

Key Words: weighted nonlocal problem, nonlinear fractional differential equation, Reimann Liouville integral and derivative, fractional differential equation, fractional integral equation
\end{abstract}

Received: March 11, 2016

Published: May 31, 2016

${ }^{\S}$ Correspondence author (c) 2016 Academic Publications, Ltd. url: www.acadpubl.eu 


\section{Introduction}

Fractional Calculus is the branch of mathematics related to the derivatives and integral of integer as well as noninteger order. This branch of mathematics has been applied to almost every field of Mathematics, Science, Engineering and Technology etc.

Nonlinear fractional differential equation with weighted initial data has been studied by several authors. The weighted Cauchy-type problem

$$
\begin{array}{r}
D^{\alpha}(u(t))=f(t, u(t)), \\
\left.t^{1-\alpha} u(t)\right|_{t=0}=b,
\end{array}
$$

studied by Khaled et al in [9].

The solution of the periodic boundary value problem for a fractional differential equation involving a RiemannLiouville fractional derivative

$$
\begin{aligned}
& D^{\alpha}(u(t))=f(t, u(t)), \\
& \left.t^{1-\alpha} u(t)\right|_{t=0}=\left.t^{1-\alpha} u(t)\right|_{t=T},
\end{aligned}
$$

studied by Weia et al in [10]. Also the existence of solutions of fractional equations of Volterra type with the RiemannLiouville derivative,

$$
\begin{aligned}
& D^{\alpha}(u(t))=f\left(t, u(t), \int_{0}^{t} k(t, s) u(s) d s\right) \\
& \left.t^{1-\alpha} u(t)\right|_{t=0}=r
\end{aligned}
$$

Studied by Jankowski [11] etc. and the references therein.Problems in nonlinear fractional differential equation were studied by various researchers[12-18]. Now here we consider the weighted nonlocal fractional differential equation

$$
\begin{aligned}
& A_{0} v(t)+\sum_{i=1}^{n} A_{i} D^{\beta_{i}}(v(t) u(t))=D^{\alpha}(v(t) u(t)), \\
& \lim _{t \rightarrow 0^{+}} t^{1-\alpha} v(t) u(t)=\sum_{j=1}^{m} a_{j} u\left(\tau_{j}\right),
\end{aligned}
$$

where $D^{\alpha}$ and $D^{\beta_{i}}$ are Riemann Liouville fractional derivatives of order $0<$ $\alpha, \beta_{i} \leq 1$ and $0<t, \tau_{j} \leq T<\infty$. 


\section{Auxiliary Results}

We will use the following definitions, lemmas and theorems in the next sections.

The extensively studied fractional derivative and integral are RiemannLiouville fractional derivative and integral, defined by [1]-[7].

Definition 2.1. The Riemann-Liouville fractional integral of order $\alpha$ of a real valued function $f$ is defined by

$$
I_{a}^{\alpha} f(z)=\frac{1}{\Gamma \alpha} \int_{a}^{z}(z-t)^{\alpha-1} f(t) d t
$$

and when $a=0$ this becomes

$$
I^{\alpha} f(z)=\frac{1}{\Gamma \alpha} \int_{0}^{z}(z-t)^{\alpha-1} f(t) d t
$$

The property studied by T.L. Holambe and Mohammed Mazhar-Ul-Haque (see [8]) is: Let $\alpha>0, \beta>0$ and $f$ be any function. Then

$$
\begin{gathered}
I_{k}^{\alpha}\left(I_{k}^{\beta} f(z)\right)=I_{k}^{\beta}\left(I_{k}^{\alpha} f(z)\right) \\
I_{k}^{\alpha}\left(I_{k}^{\beta} f(z)\right)=I_{k}^{\beta}\left(I_{k}^{\alpha} f(z)\right)=I_{k}^{\alpha+\beta} f(z) \\
=\frac{1}{k \Gamma_{k}(\alpha+\beta)} \int_{0}^{z}(z-t)^{\frac{\alpha+\beta}{k}-1} f(t) d t,
\end{gathered}
$$

for the $k$-generalized fractional integral.

Definition 2.2. The Riemann-Liouville fractional derivative of order $0<$ $\alpha \leq 1$ of a real valued function $f$ is defined by

$$
D^{\alpha} f(z)=\frac{d}{d z} I^{1-\alpha} f(z)=\frac{1}{\Gamma(1-\alpha)} \frac{d}{d z} \int_{0}^{z}(z-t)^{-\alpha} f(t) d t .
$$

Definition 2.3. A real valued function $f$ from $I \times \mathbb{R}$ is called Caratheodary function if:

1. $f$ is measurable on $\mathbb{R}$.

2. $f$ is continuous on $I$.

3. There exist Lebesgue function $h$ on $I$ such that $h$ is an Upper bound of $f$ on $I$. 
Theorem 2.4. (Kolmogorov Compactness Criterion, see [19]) Let $\Omega \subseteq$ $L^{p}(0, T), 1 \leq p<\infty$ if:

(i) $\Omega$ is bounded in $L^{p}(0, T)$, and

(ii) $u_{h} \rightarrow u$ as $h \rightarrow 0$ uniformly with respect to $u \in \Omega$,

then $\Omega$ is relatively compact in $L^{p}(0, T)$ where $u_{h}(t)=\frac{1}{h} \int_{t}^{t+h} u(s) d s$.

\section{Main Result}

For the fractional differential equation (4) with nonlocal condition we will use the following assumptions:

1. The function from $(0, T] \times \mathbb{R}$ to $\mathbb{R}$ is Caratheodary function.

2. $v(t)$ is positive and continuous with $i n f_{(0, T]}|v(t)|=v$.

3. $\sum_{j}^{m} \frac{a_{j}}{\tau_{j}^{1-\alpha} v\left(\tau_{j}\right)} \neq 1$.

Theorem 3.1. The solution of nonlocal weighted fractional differential equation (4)

$$
\begin{aligned}
& A_{0} v(t)+\sum_{i=1}^{n} A_{i} D^{\beta_{i}}(v(t) u(t))=D^{\alpha}(v(t) u(t)), \\
& \lim _{t \rightarrow 0^{+}} t^{1-\alpha} v(t) u(t)=\sum_{j=1}^{m} a_{j} u\left(\tau_{j}\right),
\end{aligned}
$$

$0<\alpha, \beta_{i} \leq 1$ and $0<t, \tau_{j} \leq T<\infty$ can be considered as

$$
\begin{aligned}
u(t)= & \frac{K t^{\alpha-1}}{v(t)} \sum_{j=1}^{m} \frac{a_{j} A_{0}}{v\left(\tau_{j}\right) \Gamma(\alpha)} \int_{0}^{\tau_{j}}\left(\tau_{j}-s\right)^{\alpha-1} v(s) d s \\
& +\frac{K t^{\alpha-1}}{v(t)} \sum_{j=1}^{m} \sum_{i=1}^{n} \frac{a_{j}}{v\left(\tau_{j}\right)} \frac{A_{i}}{\Gamma\left(\alpha-\beta_{i}\right)} \int_{0}^{\tau_{j}}\left(\tau_{j}-s\right)^{\alpha-\beta_{i}-1}(v(s) u(s)) d s \\
& +A_{0} \frac{1}{v(t) \Gamma(\alpha)} \int_{0}^{t}(t-s)^{\alpha-1} v(s) d s \\
& +\frac{1}{v(t)} \sum_{i=1}^{n} A_{i} \frac{1}{\Gamma\left(\alpha-\beta_{i}\right)} \int_{0}^{t}(t-s)^{\alpha-\beta_{i}-1}(v(s) u(s)) d s
\end{aligned}
$$


where $K=\left(1-\sum_{j=1}^{m} \frac{a_{j}}{\tau_{j}^{1-\alpha} v\left(\tau_{j}\right)}\right)^{-1}$.

Proof. Let the non-local weighted fraction differential equation 4

$$
\begin{aligned}
& A_{0} v(t)+\sum_{i=1}^{n} A_{i} D^{\beta_{i}}(v(t) u(t))=D^{\alpha}(v(t) u(t)), \\
& \lim _{t \rightarrow 0^{+}} t^{1-\alpha} v(t) u(t)=\sum_{j=1}^{m} a_{j} u\left(\tau_{j}\right),
\end{aligned}
$$

$0<\alpha, \beta_{i} \leq 1$ and $0<t, \tau_{j} \leq T<\infty$.

By the Reimann Liouville fractional derivative of order $\alpha>0$ :

$$
D_{a}^{\alpha} f(z)=\frac{1}{\Gamma(n-\alpha)} \frac{d^{n}}{d z^{n}} \int_{a}^{z}(z-t)^{n-\alpha-1} f(t) d t, \quad n-1<\alpha \leq n, n \in \mathbb{N},
$$

for $n=1, a=0$ :

$$
D^{\alpha} f(z)=\frac{1}{\Gamma(1-\alpha)} \frac{d}{d z} \int_{0}^{z}(z-t)^{-\alpha} f(t) d t .
$$

By the definition of Reimann liouville fractional integral

$$
D^{\alpha} f(z)=\frac{d}{d z} I^{1-\alpha} f(z)
$$

we obtain

$$
\begin{gathered}
A_{0} v(t)+\sum_{i=1}^{n} A_{i} D^{\beta_{i}}(v(t) u(t))=D^{\alpha}(v(t) u(t)) \\
A_{0} v(t)+\sum_{i=1}^{n} A_{i} \frac{d}{d t} I^{1-\beta_{i}}(v(t) u(t))=\frac{d}{d t} I^{1-\alpha}(v(t) u(t)) .
\end{gathered}
$$

Integrating both side from 0 to $t$, we receive

$$
\begin{aligned}
& A_{0} \int_{0}^{t} v(s) d s+\sum_{i=1}^{n} A_{i} I^{1-\beta_{i}}(v(t) u(t))-\left.\sum_{i=1}^{n} A_{i} I^{1-\beta_{i}}(v(t) u(t))\right|_{t=0} \\
&=I^{1-\alpha}(v(t) u(t))-\left.I^{1-\alpha}(v(t) u(t))\right|_{t=0} \\
& A_{0} \int_{0}^{t} v(s) d s+\sum_{i=1}^{n} A_{i} I^{1-\beta_{i}}(v(t) u(t))-C_{1}=I^{1-\alpha}(v(t) u(t))-C_{2}
\end{aligned}
$$




$$
A_{0} \int_{0}^{t} v(s) d s+\sum_{i=1}^{n} A_{i} I^{1-\beta_{i}}(v(t) u(t))+C=I^{1-\alpha}(v(t) u(t)) .
$$

Using Reimann Liouville integral $I^{\alpha}$ on bothside

$$
\begin{aligned}
& A_{0} I^{\alpha+1} v(t)+\sum_{i=1}^{n} A_{i} I^{\alpha} I^{1-\beta_{i}}(v(t) u(t))+\frac{C t^{\alpha}}{\Gamma(\alpha+1)}=I(v(t) u(t)), \\
& A_{0} I^{\alpha+1} v(t)+\sum_{i=1}^{n} A_{i} I^{\alpha-\beta_{i}+1}(v(t) u(t))+\frac{C t^{\alpha}}{\Gamma(\alpha+1)}=I(v(t) u(t)) .
\end{aligned}
$$

Taking differentiation on both side

$$
\begin{aligned}
A_{0} I^{\alpha} v(t)+\sum_{i=1}^{n} A_{i} I^{\alpha-\beta_{i}}(v(t) u(t))+\frac{C t^{\alpha-1}}{\Gamma(\alpha)} & =(v(t) u(t)), \\
t^{1-\alpha} A_{0} I^{\alpha} v(t)+t^{1-\alpha} \sum_{i=1}^{n} A_{i} I^{\alpha-\beta_{i}}(v(t) u(t))+\frac{C}{\Gamma(\alpha)} & =t^{1-\alpha}(v(t) u(t)), \\
\frac{C}{\Gamma(\alpha)} & =\lim _{t \rightarrow 0^{+}} t^{1-\alpha}(v(t) u(t)),
\end{aligned}
$$

since, by the equation (4), we have

$$
\begin{aligned}
\lim _{t \rightarrow 0^{+}} t^{1-\alpha}(v(t) u(t)) & =\sum_{j=1}^{m} a_{j} u\left(\tau_{j}\right), \\
\frac{C}{\Gamma(\alpha)} & =\sum_{j=1}^{m} a_{j} u\left(\tau_{j}\right) .
\end{aligned}
$$

Setting $t=\tau_{j}$ in equation (11), we obtain

$$
\begin{aligned}
& \tau_{j}^{1-\alpha} A_{0} \frac{1}{\Gamma(\alpha)} \int_{0}^{\tau_{j}}\left(\tau_{j}-s\right)^{\alpha-1} v(s) d s \\
&+\tau_{j}^{1-\alpha} \sum_{i=1}^{n} A_{i} \frac{1}{\Gamma\left(\alpha-\beta_{i}\right)} \int_{0}^{\tau_{j}}\left(\tau_{j}-s\right)^{\alpha-\beta_{i}-1}(v(s) u(s)) d s+\frac{C}{\Gamma(\alpha)} \\
&=\tau_{j}^{1-\alpha}\left(v\left(\tau_{j}\right) u\left(\tau_{j}\right)\right)
\end{aligned}
$$

$$
\sum_{j=1}^{m} \frac{a_{j} A_{0}}{v\left(\tau_{j}\right) \Gamma(\alpha)} \int_{0}^{\tau_{j}}\left(\tau_{j}-s\right)^{\alpha-1} v(s) d s
$$




$$
\begin{aligned}
& +\sum_{j=1}^{m} \frac{a_{j}}{v\left(\tau_{j}\right)} \sum_{i=1}^{n} \frac{A_{i}}{\Gamma\left(\alpha-\beta_{i}\right)} \int_{0}^{\tau_{j}}\left(\tau_{j}-s\right)^{\alpha-\beta_{i}-1}(v(s) u(s)) d s \\
& \left.+\sum_{j=1}^{m} \frac{C a_{j}}{\Gamma(\alpha) \tau_{j}^{1-\alpha} v\left(\tau_{j}\right)}=\sum_{j=1}^{m} a_{j} u\left(\tau_{j}\right)\right) \\
& \sum_{j=1}^{m} \frac{a_{j} A_{0}}{v\left(\tau_{j}\right) \Gamma(\alpha)} \int_{0}^{\tau_{j}}\left(\tau_{j}-s\right)^{\alpha-1} v(s) d s \\
& +\sum_{j=1}^{m} \sum_{i=1}^{n} \frac{a_{j}}{v\left(\tau_{j}\right)} \frac{A_{i}}{\Gamma\left(\alpha-\beta_{i}\right)} \int_{0}^{\tau_{j}}\left(\tau_{j}-s\right)^{\alpha-\beta_{i}-1}(v(s) u(s)) d s \\
& +\sum_{j=1}^{m} \frac{C a_{j}}{\Gamma(\alpha) \tau_{j}^{1-\alpha} v\left(\tau_{j}\right)}=\frac{C}{\Gamma(\alpha)} \\
& \sum_{j=1}^{m} \frac{a_{j} A_{0}}{v\left(\tau_{j}\right) \Gamma(\alpha)} \int_{0}^{\tau_{j}}\left(\tau_{j}-s\right)^{\alpha-1} v(s) d s \\
& +\sum_{j=1}^{m} \sum_{i=1}^{n} \frac{a_{j}}{v\left(\tau_{j}\right)} \frac{A_{i}}{\Gamma\left(\alpha-\beta_{i}\right)} \int_{0}^{\tau_{j}}\left(\tau_{j}-s\right)^{\alpha-\beta_{i}-1}(v(s) u(s)) d s \\
& =\frac{C}{\Gamma(\alpha)}-\sum_{j=1}^{m} \frac{C a_{j}}{\Gamma(\alpha) \tau_{j}^{1-\alpha} v\left(\tau_{j}\right)} \\
& \sum_{j=1}^{m} \frac{a_{j} A_{0}}{v\left(\tau_{j}\right) \Gamma(\alpha)} \int_{0}^{\tau_{j}}\left(\tau_{j}-s\right)^{\alpha-1} v(s) d s \\
& +\sum_{j=1}^{m} \sum_{i=1}^{n} \frac{a_{j}}{v\left(\tau_{j}\right)} \frac{A_{i}}{\Gamma\left(\alpha-\beta_{i}\right)} \int_{0}^{\tau_{j}}\left(\tau_{j}-s\right)^{\alpha-\beta_{i}-1}(v(s) u(s)) d s \\
& =\frac{C}{\Gamma(\alpha)}\left(1-\sum_{j=1}^{m} \frac{a_{j}}{\tau_{j}^{1-\alpha} v\left(\tau_{j}\right)}\right) \\
& K \sum_{j=1}^{m} \frac{a_{j} A_{0}}{v\left(\tau_{j}\right) \Gamma(\alpha)} \int_{0}^{\tau_{j}}\left(\tau_{j}-s\right)^{\alpha-1} v(s) d s
\end{aligned}
$$




$$
+K \sum_{j=1}^{m} \sum_{i=1}^{n} \frac{a_{j}}{v\left(\tau_{j}\right)} \frac{A_{i}}{\Gamma\left(\alpha-\beta_{i}\right)} \int_{0}^{\tau_{j}}\left(\tau_{j}-s\right)^{\alpha-\beta_{i}-1}(v(s) u(s)) d s=\frac{C}{\Gamma(\alpha)}
$$

where $K=\left(1-\sum_{j=1}^{m} \frac{a_{j}}{\tau_{j}^{1-\alpha} v\left(\tau_{j}\right)}\right)^{-1}$.

Thus, the solution from equation (11) is

$$
\begin{aligned}
u(t)= & \frac{K t^{\alpha-1}}{v(t)} \sum_{j=1}^{m} \frac{a_{j} A_{0}}{v\left(\tau_{j}\right) \Gamma(\alpha)} \int_{0}^{\tau_{j}}\left(\tau_{j}-s\right)^{\alpha-1} v(s) d s \\
& +\frac{K t^{\alpha-1}}{v(t)} \sum_{j=1}^{m} \sum_{i=1}^{n} \frac{a_{j}}{v\left(\tau_{j}\right)} \frac{A_{i}}{\Gamma\left(\alpha-\beta_{i}\right)} \int_{0}^{\tau_{j}}\left(\tau_{j}-s\right)^{\alpha-\beta_{i}-1}(v(s) u(s)) d s \\
& +A_{0} \frac{1}{v(t) \Gamma(\alpha)} \int_{0}^{t}(t-s)^{\alpha-1} v(s) d s \\
& +\frac{1}{v(t)} \sum_{i=1}^{n} A_{i} \frac{1}{\Gamma\left(\alpha-\beta_{i}\right)} \int_{0}^{t}(t-s)^{\alpha-\beta_{i}-1}(v(s) u(s)) d s
\end{aligned}
$$

Theorem 3.2. Let the assumptions 1-3 are satisfied by the non-local problem (4)

$$
\begin{aligned}
& A_{0} v(t)+\sum_{i=1}^{n} A_{i} D^{\beta_{i}}(v(t) u(t))=D^{\alpha}(v(t) u(t)), \\
& \lim _{t \rightarrow 0^{+}} t^{1-\alpha} v(t) u(t)=\sum_{j=1}^{m} a_{j} u\left(\tau_{j}\right), \\
& 0<\alpha, \beta_{i} \leq 1 \text { and } 0<t, \tau_{j} \leq T<\infty .
\end{aligned}
$$

Then the non-local problem has atleast one $L_{1}$ solution.

Proof. Let $T$ be an operator defined by

$$
\begin{aligned}
(T u)(t)= & \frac{K t^{\alpha-1}}{v(t)} \sum_{j=1}^{m} \frac{a_{j} A_{0}}{v\left(\tau_{j}\right) \Gamma(\alpha)} \int_{0}^{\tau_{j}}\left(\tau_{j}-s\right)^{\alpha-1} v(s) d s \\
& +\frac{K t^{\alpha-1}}{v(t)} \sum_{j=1}^{m} \sum_{i=1}^{n} \frac{a_{j}}{v\left(\tau_{j}\right)} \frac{A_{i}}{\Gamma\left(\alpha-\beta_{i}\right)} \int_{0}^{\tau_{j}}\left(\tau_{j}-s\right)^{\alpha-\beta_{i}-1}(v(s) u(s)) d s \\
& +A_{0} \frac{1}{v(t) \Gamma(\alpha)} \int_{0}^{t}(t-s)^{\alpha-1} v(s) d s \\
& +\frac{1}{v(t)} \sum_{i=1}^{n} A_{i} \frac{1}{\Gamma\left(\alpha-\beta_{i}\right)} \int_{0}^{t}(t-s)^{\alpha-\beta_{i}-1}(v(s) u(s)) d s .
\end{aligned}
$$


We can write it as,

$$
\begin{aligned}
(T u)(t)= & \frac{K t^{\alpha-1}}{v(t)} \sum_{j=1}^{m} \frac{a_{j}}{v\left(\tau_{j}\right)}\left\{A_{0} I^{\alpha} v\left(\tau_{j}\right)+\sum_{i=1}^{n} A_{i} I^{\alpha-\beta_{i}}\left(v\left(\tau_{j}\right) u\left(\tau_{j}\right)\right)\right\} \\
& +\frac{A_{0}}{v(t)} I^{\alpha} v(t)+\frac{1}{v(t)} \sum_{i=1}^{n} A_{i} I^{\alpha-\beta_{i}}(v(t) u(t)) .
\end{aligned}
$$

Let $\eta<\min \left\{\alpha, \alpha-\beta_{i}\right\}$, then we receive

$$
\begin{aligned}
& (T u)(t)=\frac{K t^{\alpha-1}}{v(t)} \sum_{j=1}^{m} \frac{a_{j}}{v\left(\tau_{j}\right)}\left\{A_{0} I^{\alpha-\eta} I^{\eta} v\left(\tau_{j}\right)+\sum_{i=1}^{n} A_{i} I^{\alpha-\beta_{i}-\eta} I^{\eta}\left(v\left(\tau_{j}\right) u\left(\tau_{j}\right)\right)\right\} \\
& \quad+\frac{A_{0}}{v(t)} I^{\alpha-\eta} I^{\eta} v(t)+\frac{1}{v(t)} \sum_{i=1}^{n} A_{i} I^{\alpha-\beta_{i}-\eta} I^{\eta}(v(t) u(t)) \\
& |(T u)(t)| \leq \frac{|K| t^{\alpha-1}}{i n f|v(t)|} \sum_{j=1}^{m} \frac{\left|a_{j}\right|}{i n f\left|v\left(\tau_{j}\right)\right|}\left\{\left|A_{0}\right| I^{\alpha-\eta} I^{\eta}\left|v\left(\tau_{j}\right)\right|\right. \\
& \left.+\sum_{i=1}^{n}\left|A_{i}\right| I^{\alpha-\beta_{i}-\eta} I^{\eta}\left|\left(v\left(\tau_{j}\right) u\left(\tau_{j}\right)\right)\right|\right\} \\
& \quad+\frac{\left|A_{0}\right|}{i n f|v(t)|} I^{\alpha-\eta} I^{\eta}|v(t)| \\
& \quad \frac{1}{i n f|v(t)|} \sum_{i=1}^{n}\left|A_{i}\right| I^{\alpha-\beta_{i}-\eta} I^{\eta}|(v(t) u(t))| .
\end{aligned}
$$

Let $M=\max _{I}\left\{I^{\eta} v(t) u(t)\right\}$, then

$$
\begin{aligned}
|(T u)(t)| \leq & \frac{|K| t^{\alpha-1}}{i n f|v(t)|} \sum_{j=1}^{m} \frac{\left|a_{j}\right|}{i n f\left|v\left(\tau_{j}\right)\right|} \\
& \left\{\left|A_{0}\right| M \int_{0}^{\tau_{j}} \frac{\left(\tau_{j}-s\right)^{\alpha-\eta-1}}{\Gamma(\alpha-\eta)} d s\right. \\
& \left.+\sum_{i=1}^{n}\left|A_{i}\right| M \int_{0}^{\tau_{j}} \frac{\left(\tau_{j}-s\right)^{\alpha-\beta_{i}-\eta-1}}{\Gamma\left(\alpha-\beta_{i}-\eta\right.} d s\right\} \\
& +\frac{\left|A_{0}\right|}{i n f|v(t)|} M \int_{0}^{t} \frac{(t-s)^{\alpha-\eta-1}}{\Gamma(\alpha-\eta} d s \\
& +\frac{1}{i n f|v(t)|} \sum_{i=1}^{n}\left|A_{i}\right| M \int_{0}^{t} \frac{(t-s)^{\alpha-\beta_{i}-\eta-1}}{\Gamma\left(\alpha-\beta_{i}-\eta\right.} d s
\end{aligned}
$$




$$
\begin{aligned}
\leq & \frac{|K| t^{\alpha-1}}{v} \sum_{j=1}^{m} \frac{\left|a_{j}\right|}{v}\left\{\left|A_{0}\right| M \frac{\tau_{j}^{\alpha-\eta}}{\Gamma(\alpha-\eta)}+\sum_{i=1}^{n}\left|A_{i}\right| M \frac{\tau_{j}^{\alpha-\beta_{i}-\eta}}{\Gamma\left(\alpha-\beta_{i}-\eta\right)}\right\} \\
& +\frac{\left|A_{0}\right|}{v} M \frac{t^{\alpha-\eta}}{\Gamma(\alpha-\eta)}+\frac{1}{v} \sum_{i=1}^{n}\left|A_{i}\right| M \frac{t^{\alpha-\beta_{i}-\eta}}{\Gamma\left(\alpha-\beta_{i}-\eta\right)} \\
\leq & \frac{|K| t^{\alpha-1}}{v^{2}} \sum_{j=1}^{m}\left|a_{j}\right|\left\{\left|A_{0}\right| M \frac{T^{\alpha-\eta}}{\Gamma(\alpha-\eta)}+\sum_{i=1}^{n}\left|A_{i}\right| M \frac{T^{\alpha-\beta_{i}-\eta}}{\Gamma\left(\alpha-\beta_{i}-\eta\right)}\right\} \\
& +\frac{\left|A_{0}\right|}{v} M \frac{T^{\alpha-\eta}}{\Gamma(\alpha-\eta)}+\frac{1}{v} \sum_{i=1}^{n}\left|A_{i}\right| M \frac{T^{\alpha-\beta_{i}-\eta}}{\Gamma\left(\alpha-\beta_{i}-\eta\right)} \\
\leq & \left(\frac{|K| t^{\alpha-1}}{v} \sum_{j=1}^{m}\left|a_{j}\right|+1\right. \\
& \left\{\frac{\left|A_{0}\right| M}{v} \frac{T^{\alpha-\eta}}{\Gamma(\alpha-\eta)}+\sum_{i=1}^{n} \frac{\left|A_{i}\right| M}{v} \frac{T^{\alpha-\beta_{i}-\eta}}{\Gamma\left(\alpha-\beta_{i}-\eta\right)}\right\} .
\end{aligned}
$$

Therefore

$$
\begin{aligned}
\|T u\|_{L_{1}} & =\int_{0}^{T}|(T u)(t)| d t \\
\leq & \int_{0}^{T}\left(\frac{|K| t^{\alpha-1}}{v} \sum_{j=1}^{m}\left|a_{j}\right|+1 \mid\right. \\
& \left\{\frac{\left|A_{0}\right| M}{v} \frac{T^{\alpha-\eta}}{\Gamma(\alpha-\eta)}+\sum_{i=1}^{n} \frac{\left|A_{i}\right| M}{v} \frac{T^{\alpha-\beta_{i}-\eta}}{\Gamma\left(\alpha-\beta_{i}-\eta\right)}\right\} d t \\
\leq & \left\{\frac{\left|A_{0}\right| M}{v} \frac{T^{\alpha-\eta}}{\Gamma(\alpha-\eta)}+\sum_{i=1}^{n} \frac{\left|A_{i}\right| M}{v} \frac{T^{\alpha-\beta_{i}-\eta}}{\Gamma\left(\alpha-\beta_{i}-\eta\right)}\right\} \\
& \int_{0}^{T}\left(\frac{|K| t^{\alpha-1}}{v} \sum_{j=1}^{m}\left|a_{j}\right|+\left.1\right|^{d t}\right. \\
\leq & \left\{\frac{\left|A_{0}\right| M}{v} \frac{T^{\alpha-\eta}}{\Gamma(\alpha-\eta)}+\sum_{i=1}^{n} \frac{\left|A_{i}\right| M}{v} \frac{T^{\alpha-\beta_{i}-\eta}}{\Gamma\left(\alpha-\beta_{i}-\eta\right)}\right\} \\
& \left(\frac{|K| \int_{0}^{T} t^{\alpha-1} d t}{v} \sum_{j=1}^{m}\left|a_{j}\right|+\int_{0}^{T} d t \mid\right.
\end{aligned}
$$




$$
\begin{aligned}
\leq & \left\{\frac{\left|A_{0}\right| M}{v} \frac{T^{\alpha-\eta}}{\Gamma(\alpha-\eta)}+\sum_{i=1}^{n} \frac{\left|A_{i}\right| M}{v} \frac{T^{\alpha-\beta_{i}-\eta}}{\Gamma\left(\alpha-\beta_{i}-\eta\right)}\right\} \\
& \left(\frac{|K| T^{\alpha}}{v} \sum_{j=1}^{m}\left|a_{j}\right|+T \mid .\right.
\end{aligned}
$$

Let

$$
\begin{gathered}
r=\left\{\frac{\left|A_{0}\right| M}{v} \frac{T^{\alpha-\eta}}{\Gamma(\alpha-\eta)}+\sum_{i=1}^{n} \frac{\left|A_{i}\right| M}{v} \frac{T^{\alpha-\beta_{i}-\eta}}{\Gamma\left(\alpha-\beta_{i}-\eta\right)}\right\} \\
\qquad \quad\left(\frac{|K| T^{\alpha}}{v} \sum_{j=1}^{m}\left|a_{j}\right|+T\right)^{.}
\end{gathered}
$$

Define the subset $B_{r} \subset L_{1}(I)$ by $B_{r}=\left\{u(t), t \in I:\|u\|_{L_{1}} \leq r, r>0\right\}$ the set $B_{r}$ is nonempty, closed and convex. So $\|T u\|_{L_{1}} \leq r$, which implies that the operator $T$ maps $B_{r}$ into itself. Implies that $T$ is continuous. Now, to apply Theorem 2.4, we will show that $T$ is compact. So, let $Q_{r}$ be a bounded subset of $B_{r}$. Then $T\left(Q_{r}\right)$ is bounded in $L_{1}(I)$. It remains to show that $(T u)_{h} \rightarrow(T u)$ in $L_{1}(I)$ as $h \rightarrow 0$, uniformly with respect to $T u \in T\left(Q_{r}\right)$. Now, we have

$$
\begin{aligned}
& \left\|(T u)_{h}-(T u)\right\|=\int_{0}^{T}\left|(T u)_{h}-(T u)\right| d t \\
= & \int_{0}^{T}\left|\frac{1}{h} \int_{t}^{t+h}(T u)(s) d s-(T u)\right| d t \\
\leq & \int_{0}^{T}\left(\frac{1}{h} \int_{t}^{t+h}|(T u)(s)-(T u)| d s\right) d t \\
\leq & \int_{0}^{T}\left(\frac{1}{h} \int_{t}^{t+h}|(T u)(s)-(T u)| d s\right) d t \\
\leq & \int_{0}^{T} \frac{1}{h} \int_{t}^{t+h} \mid\left(\frac{K s^{\alpha-1}}{v(s)} \sum_{j=1}^{m} \frac{a_{j}}{v\left(\tau_{j}\right)}\left\{A_{0} I^{\alpha} v\left(\tau_{j}\right)+\sum_{i=1}^{n} A_{i} I^{\alpha-\beta_{i}}\left(v\left(\tau_{j}\right) u\left(\tau_{j}\right)\right)\right\}\right. \\
& \left.+\frac{A_{0}}{v(s)} I^{\alpha} v(s)+\frac{1}{v(s)} \sum_{i=1}^{n} A_{i} I^{\alpha-\beta_{i}}(v(s) u(s))\right) \\
& -\left(\frac{K t^{\alpha-1}}{v(t)} \sum_{j=1}^{m} \frac{a_{j}}{v\left(\tau_{j}\right)}\left\{A_{0} I^{\alpha} v\left(\tau_{j}\right)+\sum_{i=1}^{n} A_{i} I^{\alpha-\beta_{i}}\left(v\left(\tau_{j}\right) u\left(\tau_{j}\right)\right)\right\}\right.
\end{aligned}
$$




$$
\begin{aligned}
& \left.+\frac{A_{0}}{v(t)} I^{\alpha} v(t)+\frac{1}{v(t)} \sum_{i=1}^{n} A_{i} I^{\alpha-\beta_{i}}(v(t) u(t))\right) \mid d s d t \\
\leq & \sum_{j=1}^{m} \frac{a_{j}}{v\left(\tau_{j}\right)}\left\{A_{0} I^{\alpha} v\left(\tau_{j}\right)+\sum_{i=1}^{n} A_{i} I^{\alpha-\beta_{i}}\left(v\left(\tau_{j}\right) u\left(\tau_{j}\right)\right)\right\} \\
& \int_{0}^{T} \frac{1}{h} \int_{t}^{t+h}\left|\left(\frac{K s^{\alpha-1}}{v(s)}\right)-\left(\frac{K t^{\alpha-1}}{v(t)}\right)\right| d s d t \\
& +\int_{0}^{T} \frac{1}{h} \int_{t}^{t+h} \mid \frac{A_{0}}{v(s)} I^{\alpha} v(s)+\frac{1}{v(s)} \sum_{i=1}^{n} A_{i} I^{\alpha-\beta_{i}}(v(s) u(s)) \\
& -\frac{A_{0}}{v(t)} I^{\alpha} v(t)-\frac{1}{v(t)} \sum_{i=1}^{n} A_{i} I^{\alpha-\beta_{i}}(v(t) u(t)) \mid d s d t
\end{aligned}
$$

since:

$$
\frac{1}{h} \int_{t}^{t+h}\left|\left(\frac{K s^{\alpha-1}}{v(s)}\right)-\left(\frac{K t^{\alpha-1}}{v(t)}\right)\right| d s \rightarrow 0
$$

and

$$
\begin{aligned}
\frac{1}{h} \int_{t}^{t+h} \mid \frac{A_{0}}{v(s)} I^{\alpha} v(s)+ & \frac{1}{v(s)} \sum_{i=1}^{n} A_{i} I^{\alpha-\beta_{i}}(v(s) u(s)) \\
& \quad-\frac{A_{0}}{v(t)} I^{\alpha} v(t)-\frac{1}{v(t)} \sum_{i=1}^{n} A_{i} I^{\alpha-\beta_{i}}(v(t) u(t)) \mid d s \longrightarrow 0 .
\end{aligned}
$$

Here, we used that the sum belongs to $L_{1}(I)$ and $\frac{K t^{\alpha-1}}{v(t)} \in L_{1}(I)$.

Therefore, by Theorem 2.4, we have that $T\left(Q_{r}\right)$ is relatively compact, that is, $T$ is a compact operator, then the operator $\mathrm{T}$ has a fixed point in $B_{r}$, which proves the existence of solution in $L_{1}(I)$.

\section{References}

[1] E.D. Rain Ville, Special Functions, Macmillan, New York, 1960.

[2] K.B. Oldham, J. Spanier, The Fractional Calculus, Academic Press, New York, 1974.

[3] I. Podlubny, Fractional Differential Equations, Academic Press, San Diego, CaliforniaU.S.A., 1999.

[4] P.L. Butzer, A.A. Kilbas, J.J. Trujillo, Compositions of Hadamard-type fractional integration operators and the semigroup property, Journal of Mathematical Analysis and Applications, 269 (2002), 387-400. 
[5] P.L. Butzer, A.A. Kilbas, J.J. Trujillo, Fractional calculus in the Mellin setting and Hadamard-type fractional integrals, Journal of Mathematical Analysis and Applications, 269 (2002), 1-27.

[6] P.L. Butzer, A.A. Kilbas, J.J. Trujillo, Mellin transform analysis and integration by parts for Hadamard-type fractional integrals, Journal of Mathematical Analysis and Applications, 270 (2002), 1-15.

[7] A. Kilbas, Hadamard-type Fractional Calculus, Journal of Korean Mathematical Society, 38, No. 6 (2001), 1191-1204.

[8] T.L. Holambe, Mohammed Mazhar-Ul-Haque, A remark on semi group property in fractional calculus, International Journal of Mathematics and Computer Application Research, 4, No. 6 (2014), 27-32.

[9] M. Khaled, M. Furati, N.E. Tatar, Power-type estimates for nonlinear fractional differential equation, Nonlinear Anal., 62 (2005), 10251036.

[10] Zhongli Weia, Wei Dongc, Junling Chea, Periodic boundary value problems for fractional differential equations involving a RiemannLiouville fractional derivative, Nonlinear Anal., 73 (2010), 32323238.

[11] T. Jankowski, Fractional equations of Volterra type involving a RiemannLiouville derivative, Appl. Math. Lett., 26 (2013), 344350.

[12] F.M. Gaffar Continuous and integrable solutions of a nonlinear Cauchy problem of fractional order with nonlocal conditions, Journal of the Egyptian Mathematical Society, 22 (2014), 341347.

[13] A.M.A. El-Sayed, E.O. Bin-Taher, An arbitrary (fractional) orders differential equation with internal nonlocal and integral conditions, Adv. Pure Math., 1 (2011), 5962.

[14] A.M.A. El-Sayed, E.O. Bin-Taher, A nonlocal problem for a multiterm fractional-order differential equation, Int. J. Math. Anal., 5,. No. 29 (2011), 14451451.

[15] A.M.A. El-Sayed, E.O. Bin-Taher, Maximal and minimal positive solutions for a nonlocal boundary value problem of a fractional-order differential equation, J. Fract. Calc. Appl., 1, No. 7 (2011) 18.

[16] A. Boucherif, First-order differential inclusions with nonlocal initial conditions, Appl. Math. Lett., 15 (2002), 409414.

[17] A. Boucherif, Semilinear evolution inclusions with nonlocal conditions, Appl. Math. Lett., 22 (2009), 11451149.

[18] Zhang Shuqin, Monotone iterative method for initial value problem involving RiemannLiouville fractional derivatives, Nonlinear Anal., 71 (2009), 20872093.

[19] J. Dugundji, A. Granas, Fixed Point Theory, Monografie Mathematyczne, PWN, Warsaw (1982). 
